\title{
Influence of Preoperative Anti-TNF-Alpha Antibody Therapy on Postoperative Recurrence of Crohn's Disease
}

\section{Takashi Takeda}

Department of Gastroenterological Surgery, Graduate School of Medicine, Osaka University Tsunekazu Mizushima ( $\sim$ tmizushima@gesurg.med.osaka-u.ac.jp )

Department of Gastroenterological Surgery, Graduate School of Medicine, Osaka University

\section{Terukazu Yoshihara}

Department of Gastroenterological Surgery, Graduate School of Medicine, Osaka University

\section{Takayuki Ogino}

Department of Gastroenterological Surgery, Graduate School of Medicine, Osaka University

\section{Shiki Fujino}

Department of Gastroenterological Surgery, Graduate School of Medicine, Osaka University

\section{Tsuyoshi Hata}

Department of Gastroenterological Surgery, Graduate School of Medicine, Osaka University Norikatsu Miyoshi

Department of Gastroenterological Surgery, Graduate School of Medicine, Osaka University Hidekazu Takahashi

Department of Gastroenterological Surgery, Graduate School of Medicine, Osaka University

\section{Mamoru Uemura}

Department of Gastroenterological Surgery, Graduate School of Medicine, Osaka University Hirofumi Yamamoto

Department of Gastroenterological Surgery, Graduate School of Medicine, Osaka University

\section{Yuichiro Doki}

Department of Gastroenterological Surgery, Graduate School of Medicine, Osaka University Hidetoshi Eguchi

Department of Gastroenterological Surgery, Graduate School of Medicine, Osaka University

\section{Research Article}

Keywords: Crohn's disease, Anti-TNF alpha, Postoperative recurrence, Loss of response

Posted Date: February 12th, 2021

DOl: https://doi.org/10.21203/rs.3.rs-172713/v1 
License: (c) (i) This work is licensed under a Creative Commons Attribution 4.0 International License. Read Full License 


\section{Abstract}

Background: Anti-TNFa antibody is effective for controlling inflammation caused by Crohn's disease. However, an increasing number of patients have recently undergone surgery after loss of response during maintenance therapy with anti-TNFa antibody.

Aims: The purpose of this study was to examine how preoperative treatment using anti-TNFa antibody affects postoperative recurrence.

Methods: Between January 2002 and June 2020, we retrospectively analyzed 90 patients with Crohn's disease who underwent bowel resection with anastomosis and received endoscopic evaluation within 18 months after surgery.

Results: Fifty-seven patients had used anti-TNF antibodies preoperatively, and 33 had not. Of the 57 patients, 31 underwent surgery after loss of response. At the time of the first postoperative endoscopy, endoscopic recurrence occurred in 20 patients (35.1\%) who used anti-TNFa antibody and 5 patients $(15.2 \%)$ who did not use anti-TNFa antibody $(p=0.0419)$. The median symptomatic recurrence-free duration was 8 months in patients with loss of response and 44 months in patients without a loss of response $(p=0.0108)$.

Conclusions: Patients who received anti-TNFa antibody preoperatively were prone to endoscopic recurrence. In addition, patients who experienced inefficacy before their surgeries were more likely to have recurrence, even after resuming post-operative anti-TNFa antibody treatment.

\section{Introduction}

Crohn's disease (CD) is a chronic inflammatory bowel disease that is common among young people from their late teens to early 20s. In recent years, the number of patients with CD has increased not only in Europe and the United States, but also in Asia, including Japan [1, 2]. In 2014, the estimated numbers of patients with CD in Japan is 70,700 [3]. Although the etiology is currently not known, it is thought to be a multifactorial disease that develops through abnormal immune responses with the addition of environmental and genetic factors. Many patients require surgery during the course of $C D$. The cumulative surgery rate for $C D$ within 10 years after diagnosis is approximately $44-50 \%$ [4]. The postoperative recurrence rate is high, and how well medical treatment controls inflammation of the intestinal tract is important [5]. Surgery is based on minimal resection of adaptive lesions and strictureplasty [6], but in the long term, complications due to short bowel syndrome and colostomy become problematic. Treatment strategies that prevent repeat surgeries are important to improve the prognosis of $\mathrm{CD}[7]$.

Medications such as infliximab and adalimumab, which are antibodies against TNFa, an inflammatory cytokine, can be used for CD patients [8, 9]. In addition, anti-IL-12/23p40 monoclonal antibody 
ustekinumab, Janus kinase (JAK) inhibitors, and monoclonal antibody targeting the a4 $\beta 7$ integrin heterodimer have been developed and are used to induce remission and for maintenance of CD [10,11].

Infliximab is started within 4 weeks after surgery in patients with CD who had undergone ileocecal resection and suffered no residual lesions, whereas maintenance therapy is performed for 1 year, with endoscopic recurrence significantly suppressed after 1 year [12]. The POCER study, examining the usefulness of endoscopy in postoperative recurrence and the effectiveness of step-up treatment for early mucosal lesions, reported that the adalimumab group had significantly suppressed recurrence compared to the thiopurine group [13]. Also, planned postoperative adalimumab maintenance therapy could be beneficial for Japanese patients with CD [14]. These results suggest that the anti-TNFa antibody may be effective in preventing postoperative recurrence. However, whether similar effects occur in cases of loss of response (LOR), a diminishing effect during maintenance therapy before surgery, is unclear. Few studies have analyzed the relationship between preoperative anti-TNFa antibody treatment history and postoperative recurrence. The purpose of this study was to investigate the relationship between preoperative treatment using anti-TNFa antibody and postoperative recurrence.

\section{Patients And Methods}

\section{Patients}

Of 155 people who underwent surgery for CD intestinal lesions for indications other than malignant tumors from January 2002 to June 2020, 90 patients who underwent lower gastrointestinal endoscopy, ileocolonoscopy, and/or double-balloon endoscopy (within 18 months after the surgeries) were included in our study analysis. All subjects were treated and followed up at Osaka University Hospital.

\section{Assessment of clinical features}

Medical data on gender, age at the time of surgery, age at the time of diagnosis, BMI, surgery history, lesion sites, blood test results, and CD treatments were collected from treatment records.

Infliximab, when used as CD treatment in Japan, is usually administered as a single intravenous infusion of $5 \mathrm{mg}$ per $1 \mathrm{~kg}$ of body weight, then 2 weeks and 6 weeks after the first dose, and then at 8-week intervals. For adalimumab, $160 \mathrm{mg}$ is usually injected subcutaneously for the first dose and $80 \mathrm{mg}$ subcutaneously 2 weeks later, and then $40 \mathrm{mg}$ subcutaneously every 2 weeks thereafter. Patients with a poor response to infliximab are given double doses, a shortened dosing interval every 4-7 weeks, or switched to adalimumab. Patients who have a poor response to adalimumab are given double doses or switched to infliximab.

First, patients were divided into two groups in order to evaluate the relationship between having or not having a preoperative anti-TNFa antibody history and postoperative recurrence. Patients who were using anti-TNFa antibody prior to surgery were defined as the TNFa group, and those who were not using it were 
defined as the non-TNFa group. In addition, the TNFa group was divided into two groups in order to evaluate the relationship between LOR due to the use of anti-TNFa antibody before surgery and recurrence after surgery. The patients who received standard doses of anti-TNFa antibody before surgery were defined as the non-LOR group, whereas the patients who received increased doses of anti-TNFa antibody and those who had their dosage adjusted were defined as the LOR group (Fig. 1). Ustekinumab, an anti-IL-12/23p40 monoclonal antibody, and JAK inhibitors were not used before surgery. The postoperative treatment policy of our department is for the non-TNFa group to have no prophylactic administration of anti-TNFa antibody, whereas the LOR group and non-LOR group both receive the same standard dose before the surgeries; thus, the treatment was strengthened according to the results of the first postoperative endoscopy.

\section{Postoperative follow-up and diagnosis of recurrence}

The patients were followed up as outpatients once every 1-3 months. Even when there were no symptoms, lower endoscopy, ileocolonoscopy, and/or double-balloon endoscopy was recommended 6 to 12 months after surgery. Endoscopy was then recommended every 1-2 years. The Crohn's Disease Activity Index (CDAl) was used to evaluate symptomatic recurrence and the Rutgeerts' score (RS) to evaluate endoscopic recurrence $[15,16]$. Symptomatic recurrence was defined as CDAI $\geq 220$ and endoscopic recurrence as $\mathrm{RS} \geq \mathrm{i} 2$.

\section{Statistical analysis}

Categorical data were compared using the chi-squared test or Fisher exact test. The Kaplan-Meier method and log rank test were used to compare symptomatic and endoscopic recurrence in the two groups and to calculate significant differences. $P<0.05$ was considered significant. All statistical analyses were performed using JMP statistical software, package 14.0 (SAS Institute Inc., Cary, NC, USA).

\section{Results}

\section{Patient characteristics}

Of the 155 patients who underwent surgery for intestinal lesions caused by CD for indications other than malignant tumors, 65 who had no endoscopic follow-up within 18 months after surgery were excluded from the analysis, and the remaining 90 people became the object of analysis. Table 1 summarizes the patient characteristics. The median age at the time of surgery was 39.5 years (67 men and 23 women). The median age at the time of diagnosis was 27.5 years. The age group at diagnosis according to the Montreal classification was as follows: A1 was 73 (81.1\%), A2 was 16 (17.8\%), and A3 was 1 (1.1\%). For disease location classification, L1 was 45 (50.0\%), L2 was 9 (10.0\%), L3 was 34 (37.8\%), and L4 was 2 (2.2\%). In the disease behavior classification of lesions, B1 was 2 (2.2\%), B2 was 35 (38.9\%), and B3 was 
53 (58.9\%). 32 people (35.6\%) were given immunomodulators, such as azathioprine and 6mercaptopurine, before surgery. Nine patients (10.0\%) received corticosteroids. 
Table 1

Baseline characteristics of Crohn's disease patients.

\section{Total}

Age, years

Male/female, $n$

Body mass index, $\mathrm{kg} / \mathrm{m}^{2}$

Age at diagnosis of Crohn's disease, years

Surgical history, primary/repeated

Age at diagnosis

A1

A2

A3

Preoperative albumin, $\mathrm{mg} / \mathrm{dL}$

Preoperative CRP, mg/dL

Preoperative CDAI

Lesion location

\section{L1}

L2

L3

L4

Disease behavior

B1

B2

B3

Preoperative medication

AZAđ6MP +/-, n

Steroid +/-, n

Anti-TNFa agent +/-, n
90

$39.5(14-72)$

$67 / 23(74.4 \% / 25.6 \%)$

$19.4 \pm 2.8$

$27.5(12-66)$

74/16 (82.2\%/17.8\%)

$73(81.1 \%)$

$16(17.8 \%)$

$1(1.1 \%)$

$3.4 \pm 0.6$

$1.4 \pm 2.7$

$188.1 \pm 109.3$

$45(50.0 \%)$

$9(10.0 \%)$

$34(37.8 \%)$

$2(2.2 \%)$

2 (2.2\%)

$35(38.9 \%)$

$53(58.9 \%)$

$32 / 58$ (35.6\%/64.4\%)

9/81 (10.0\%/90.0\%)

$57 / 33(63.3 \% / 36.7 \%)$

CRP, C-reactive protein; CDAl, Crohn's Disease Activity Index; AZA, azathioprine; 6MP, 6mercaptopurine; Anti-TNFa, anti-tumor necrosis factor a 
Data are given as median (range), mean $\pm \mathrm{SD}$, or $\mathrm{n}(\%)$

CRP, C-reactive protein; CDAl, Crohn's Disease Activity Index; AZA, azathioprine; 6MP, 6mercaptopurine; Anti-TNFa, anti-tumor necrosis factor a

\section{Clinical features of the TNF groups}

57 patients were assigned to the TNFa group and 33 to the non-TNFa group. No differences were found between the groups in regards to the age at the time of surgery, gender, BMI, age at diagnosis, surgery history, preoperative CRP values, preoperative CDAl, or use of corticosteroids. However, preoperative albumin levels were significantly lower (3.3 vs. 3.6, $p=0.0130)$ and the number of the patients using azathioprine was significantly higher $(25(43.4 \%)$ vs. $7(21.2 \%), p=0.0400)$ in the TNFa group than in the non-TNFa group (Table 2).

Table 2

Patient characteristics according to TNFa treatment group.

\begin{tabular}{|llll|}
\hline Characteristic & TNFa & Non-TNFa & P-value \\
& $\mathbf{n = 5 7}$ & $\mathbf{n = 3 3}$ & \\
\hline Age, years & $39(14-68)$ & $42(19-72)$ & 0.4586 \\
\hline Male/female, $\mathrm{n}$ & $41 / 16$ & $26 / 7$ & 0.6173 \\
\hline Body mass index, $\mathrm{kg} / \mathrm{m}^{2}$ & $19.2 \pm 2.7$ & $19.6 \pm 3.0$ & 0.5763 \\
\hline Age at diagnosis of Crohn's disease, years & $25(12-66)$ & $29(12-52)$ & 0.0516 \\
\hline Surgical history, primary/repeated & $45 / 12$ & $29 / 4$ & 0.3942 \\
\hline Preoperative Albumin, mg/dL & $3.3 \pm 0.7$ & $3.6 \pm 0.5$ & $0.0130^{\star}$ \\
\hline Preoperative CRP, mg/dL & $1.3 \pm 2.7$ & $1.5 \pm 2.8$ & 0.8179 \\
\hline Preoperative CDAl & $185.8 \pm 107.8$ & $192.2 \pm 113.6$ & 0.7958 \\
\hline Preoperative medication & & & \\
\hline AZA®6MP +/-, $\mathrm{n}$ & $25 / 32$ & $7 / 26$ & $0.0400^{*}$ \\
\hline Steroid +/-, $\mathrm{n}$ & $7 / 50$ & $2 / 31$ & 0.4771 \\
\hline Data are presented as median (range), mean \pm SD unless otherwise noted. & \\
\hline
\end{tabular}

\section{Clinical features of the LOR and non-LOR groups}


31 patients were assigned to the LOR group and 26 to the non-LOR group. No differences were found between the groups in regards to the age at the time of surgery, gender, BMI, age at diagnosis, surgery history, preoperative albumin levels, preoperative CRP values, preoperative CDAl, presence or absence of immunomodulators, or use of corticosteroids (Table 3).

Table 3

Patient characteristics according to LOR and non-LOR.

\begin{tabular}{|llll|}
\hline Characteristic & LOR & Non-LOR & P-value \\
& $\mathbf{n = 3 1}$ & $\mathbf{n = 2 6}$ & \\
\hline Age, years & $38(19-61)$ & $40(14-68)$ & 0.8670 \\
\hline Male/female, $\mathrm{n}$ & $21 / 10$ & $20 / 6$ & 0.5581 \\
\hline Body mass index, kg/m² & $19.5 \pm 2.8$ & $19.0 \pm 2.7$ & 0.4930 \\
\hline Age at diagnosis of Crohn's disease, years & $25(12-40)$ & $25.5(14-66)$ & 0.1395 \\
\hline Surgical history, primary/repeated & $26 / 5$ & $19 / 7$ & 0.3490 \\
\hline Preoperative Albumin, mg/dL & $3.3 \pm 0.6$ & $3.3 \pm 0.7$ & 0.9967 \\
\hline Preoperative CRP, mg/dL & $1.4 \pm 2.6$ & $1.3 \pm 2.9$ & 0.8397 \\
\hline Preoperative CDAl & $175.0 \pm 100.0$ & $198.7 \pm 117.1$ & 0.4201 \\
\hline Preoperative medication & & & \\
\hline AZA囚6MP +/-, $\mathrm{n}$ & $15 / 16$ & $10 / 16$ & 0.5931 \\
\hline Steroid +/-, $\mathrm{n}$ & $6 / 25$ & $1 / 25$ & 0.1118 \\
\hline Data are presented as median (range), mean \pm SD unless otherwise noted. & \\
\hline
\end{tabular}

\section{Symptomatic and endoscopic recurrence-free duration}

Figure 2 shows the symptomatic and endoscopic recurrence-free period. The median symptomatic recurrence duration was 19 months, and the median endoscopic recurrence duration was 54 months.

\section{Rate of endoscopic recurrence at first postoperative endoscopy}

Figure 3 shows the rate of endoscopic recurrence observed during the first postoperative endoscopy. The first postoperative endoscopy was performed a median 6 months (range; 1-18 months) after surgery. Of all 90 patients, 25 (27.8\%) had endoscopic recurrence. Endoscopic recurrence was observed in 20 patients (35.1\%) from the TNFa group and 5 (15.2\%) from the non-TNFa group. In the TNFa group, 9 
patients $(29.0 \%)$ from the LOR group and 11 patients (42.3\%) from the non-LOR group had endoscopic recurrence.

\section{Relationship between preoperative anti-TNFa antibody treatment history and postoperative recurrence}

The median duration of symptomatic recurrence was 11 months for the TNFa group and 40 months for the non-TNFa group $(p=0.1749)$. The median endoscopic recurrence-free duration was 21 months for the TNFa group and 60 months for the non-TNFa group ( $p=0.1311$; Fig. 4).

\section{Relationship between LOR and recurrence after surgery}

The median duration of symptomatic recurrence was 8 months for the LOR group and 44 months for the non-LOR group $(p=0.0108)$. The median endoscopic recurrence-free duration was 10 months for the LOR group and 60 months for the non-LOR group ( $p=0.0277$; Fig. 5).

\section{Discussion}

The results of $C D$ treatment have improved dramatically with advances in drug treatment, especially with the advent of biological agents[17]. With the widespread use of anti-TNFa antibody, the emergence of LOR has become a problem. Diminishing effects have been reported to occur in approximately $37 \%$ of cases [18] and are commonly seen in $20-30 \%$ of patients, but approximately $10 \%$ of patients annually and nearly half of cases in 5 years have been reported to experience LOR [19]. The main cause of LOR is a decrease in the concentration of anti-TNFa antibodies in the blood and tissues. The cause of this has been reported to be the anti-infliximab antibodies, which act against infliximab, and the anti-adalimumab antibodies, which act against adalimumab and are related to the diminishing effect [20,21]. As the main cause of LOR is a decrease in blood concentration, maintaining an adequate concentration of infliximab in the blood is important to avoid diminishing the effect during maintenance therapy with infliximab. In the ACCENT I study, in 40 cases in which a single dose of $5 \mathrm{mg} / \mathrm{kg}$ infliximab was effective after 2 weeks but diminished by week 54 , in 36 cases (90\%) the effect was recovered when the dose was increased to $10 \mathrm{mg} / \mathrm{kg}$ [22].

In addition to evaluating clinical symptoms, endoscopy is important for evaluating postoperative recurrence [23]. After ileocolic resection due to CD, only $20 \%$ of patents have symptoms 1 year after surgery, whereas $73 \%$ have recurrent lesions in the anastomosis ileum, which is observed using an endoscope [24]. Regarding recurrence after ileocolic resection, a small aphthous ulcer on the ileal side of the anastomosis appears within 1 year after surgery, and tortuous ulceration and nodular thickening occur 1 to 3 years after surgery. Stenosis at the anastomosis has been shown to occur within 3 to 10 years after surgery [25]. The evaluation of postoperative endoscopy based on these observations is the Rutgeerts' score. The symptomatic recurrence rate is low in i 0 and i1 cases, but high in i2 to i4 cases. 
In this study, we investigated the preoperative anti-TNFa antibody treatment history and postoperative recurrence. The duration to symptomatic recurrence was shorter than the duration before endoscopic recurrence. Although this contradicted the previous report, clinical symptoms were checked every 1 to 3 months during each outpatient visit, which is thought to be due to short periods of evaluation of clinical symptoms compared to intervals between endoscopies.

The TNFa group had significantly decreased serum albumin levels before surgery. Lower serum albumin concentrations significantly correlate with higher infliximab clearance, leading to shorter half-life [26]. Low serum albumin levels are also known to significantly correlate with postoperative endoscopic recurrence in patients receiving anti-TNFa antibody treatment before surgery [27], which is consistent with endoscopic recurrence being significantly more likely to occur in the TNFa group than in the non-TNFa group. In addition to low serum albumin values, male gender and high BMI are factors known to increase the clearance of anti-TNFa antibodies [28].

One of the main causes of LOR is the appearance of anti-preparation antibodies. Patients who have LOR during preoperative anti-TNFa antibody treatment are likely to have recurrence despite anti-TNFa antibody treatment after surgery due to the preoperative appearance of anti-preparation antibodies.

The current study has several limitations. First, retrospective studies conducted at a single facility cover a small number of patients. Second, the timing of endoscopy after surgery in this study varied between patients. Finally, this study failed to measure anti-drug antibodies, which is one possible cause of LOR. A new anti-TNFa antibody to replace infliximab and adalimumab, and antibody targeting cytokines and chemokines other than TNFa are currently in development. Follow-ups need to be conducted after surgeries while keeping in mind the history of preoperative antibody treatments.

In conclusion, patients who received anti-TNFa antibody treatment before surgery were prone to endoscopic recurrence. In addition, patients who experienced a loss of response before surgery were more likely to have recurrence, even after resuming anti-TNFa antibody treatment after surgery.

\section{Declarations}

\section{Ethics approval and consent to participate}

The Osaka University Clinical Research Review Committee approved this study (approval number: 150282). All patients provided written informed consent. If the patient was under 18 years of age, informed consent was obtained from the parent.

\section{Consent for publication}

Not applicable.

\section{Availability of data and materials}


The datasets generated and analysed during the current study are not publicly available due to consent from participants but are available from the corresponding author on reasonable request.

\section{Funding}

We didn't receive any specific funding for this study.

\section{Authors' contributions}

TT, TM, and TY designed the study and wrote the manuscript. TO, SF, TH, NM, HT, and MU performed data collection and analysis. HY, YD, and HE approved the manuscript. All authors read and approved the final manuscript.

\section{Acknowledgements}

We would like to thank San Francisco Edit (www.sfedit.net) for English language editing.

\section{Competing interests}

The authors declare that they have no competing interests.

\section{References}

1. Ooi CJ, Hilmi I, Banerjee R, et al. Best practices on immunomodulators and biologic agents for ulcerative colitis and Crohn's disease in Asia. Intest Res. 2019;17:285-310.

2. Ogino T, Mizushima T, Matsuda C, Mori M, Doki Y. Essential updates 2018/2019: Colorectal (benign): Recent updates (2018-2019) in the surgical treatment of benign colorectal diseases. Ann Gatroenterol Surg. 2019;4:30-38.

3. Murakami Y, Nishiwaki Y, Oba MS, et al. Estimated prevalence of ulcerative colitis and Crohn's disease in Japan in 2014: an analysis of a nationwide survey. J Gastroenterol. 2019;54:1070-1077.

4. Peyrin-Biroulet L, Loftus EV Jr, Colombel JF, Sandborn WJ. The natural history of adult Crohn's disease in populationbased cohorts. Am J Gastroenterol. 2010;105:289-297.

5. Pariente B, Cosnes J, Danese S, et al. Development of the Crohn's disease digestive damage score, the Lémann score. Inflamm Bowel Dis. 2011;17:1415-1422.

6. Alexander-Williams J, Haynes IG. Up-to-date management of small-bowel Crohn's disease. Adv Surg. 1987;20:245-264.

7. Watanabe Y, Miyoshi N, Fujino S, et al. Cumulative Inflammation Could Be a Risk Factor for Intestinal Failure in Crohn's Disease. Dig Dis Sci. 2019;64:2280-2285.

8. Targan SR, Hanauer SB, van Deventer SJ, et al. A short-term study of chimeric monoclonal antibody CA2 to tumor necrosis factor alpha for Crohn's disease. Crohn's Disease cA2 Study Group. N Engl J Med. 1997;337:1029-1035. 
9. Colombel JF, Sandborn WJ, Rutgeerts P, et al. Adalimumab for maintenance of clinical response and remission in patients with Crohn's disease: the CHARM trial. Gastroenterology. 2007;132:52-65.

10. Sandborn WJ, Gasink C, Gao LL, et al. Ustekinumab induction and maintenance therapy in refractory Crohn's disease. N Engl J Med. 2012;367:1519-1528.

11. Vermeire $S$, Schreiber $S$, Petryka R, et al. Clinical remission in patients with moderate-to-severe Crohn's disease treated with filgotinib (the FITZROY study): results from a phase 2, double-blind, randomised, placebo-controlled trial. Lancet. 2017;389:266-275.

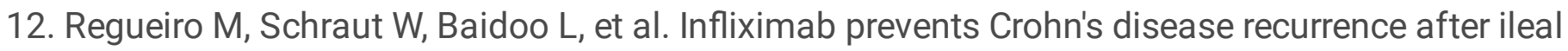
resection. Gastroenterology. 2009; 136:441-450.

13. De Cruz P, Kamm MA, Hamilton AL, et al. Crohn's disease management after intestinal resection: a randomised trial. Lancet. 2015;385:1406-1417.

14. Asada T, Nakayama G, Tanaka C, et al. Postoperative adalimumab maintenance therapy for Japanese patients with Crohn's disease: a single-center, single-arm phase II trial (CCOG-1107 study). Surg Today. 2018;48:609-617.

15. Ohara $\mathrm{N}$, Mizushima $\mathrm{T}$, lijima $\mathrm{H}$, et al. Adherence to an elemental diet for preventing postoperative recurrence of Crohn's disease. Surg Today. 2017;47:1519-1525.

16. Ikeda A, Miyoshi N, Fujino S, et al. A Novel Predictive Nomogram for Early Endoscopic Recurrence after Intestinal Resection for Crohn's Disease. Digestion. 2019;100:269-276.

17. Grevenitis P, Thomas A, Lodhia N. Medical therapy for inflammatory bowel disease. Surg Clin North Am. 2015;95:1159-1182.

18. Gisbert JP, Panés J. Loss of response and requirement of infliximab dose intensification in Crohn's disease: a review. Am J Gastroenterol. 2009;104:760-767.

19. Schnitzler F, Fidder H, Ferrante $M$, et al. Long-term outcome of treatment with infliximab in 614 patients with Crohn's disease: results from a single-centre cohort. Gut. 2009;58:492-500.

20. Nanda KS, Cheifetz AS, Moss AC. Impact of antibodies to infliximab on clinical outcomes and serum infliximab levels in patients with inflammatory bowel disease (IBD): a meta-analysis. Am J Gastroenterol. 2013;108:40-47.

21. Karmiris K, Paintaud G, Noman M, et al. Influence of trough serum levels and immunogenicity on long-term outcome of adalimumab therapy in Crohn's disease. Gastroenterology. 2009;137:16281640.

22. Rutgeerts P, Feagan BG, Lichtenstein GR, et al. Comparison of scheduled and episodic treatment strategies of infliximab in Crohn's disease. Gastroenterology. 2004;126:402-413.

23. Gionchetti P, Dignass A, Danese S, et al. 3rd European Evidence-based Consensus on the Diagnosis and Management of Crohn's Disease 2016: Part 2: Surgical Management and Special Situations. J Crohns Colitis. 2017;11:135-149.

24. Rutgeerts P, Geboes K, Vantrappen G, Beyls J, Kerremans R, Hiele M. Predictability of the postoperative course of Crohn's disease. Gastroenterology. 1990;99:956-963. 
25. Rutgeerts P, Geboes K, Vantrappen G, Kerremans R, Coenegrachts JL, Coremans G. Natural history of recurrent Crohn's disease at the ileocolonic anastomosis after curative surgery. Gut. 1984;25:665672.

26. Dotan I, Ron Y, Yanai H, et al. Patient factors that increase infliximab clearance and shorten half-life in inflammatory bowel disease: a population pharmacokinetic study. Inflamm Bowel Dis. 2014;20:2247-2259.

27. Hiraoka S, Takashima S, Kondo Y, et al. Efficacy of restarting anti-tumor necrosis factor a agents after surgery in patients with Crohn's disease. Intest Res. 2018;16:75-82.

28. Ordás I, Mould DR, Feagan BG, Sandborn WJ. Anti-TNF monoclonal antibodies in inflammatory bowel disease: pharmacokinetics-based dosing paradigms. Clin Pharmacol Ther. 2012;91:635-646.

\section{Figures}

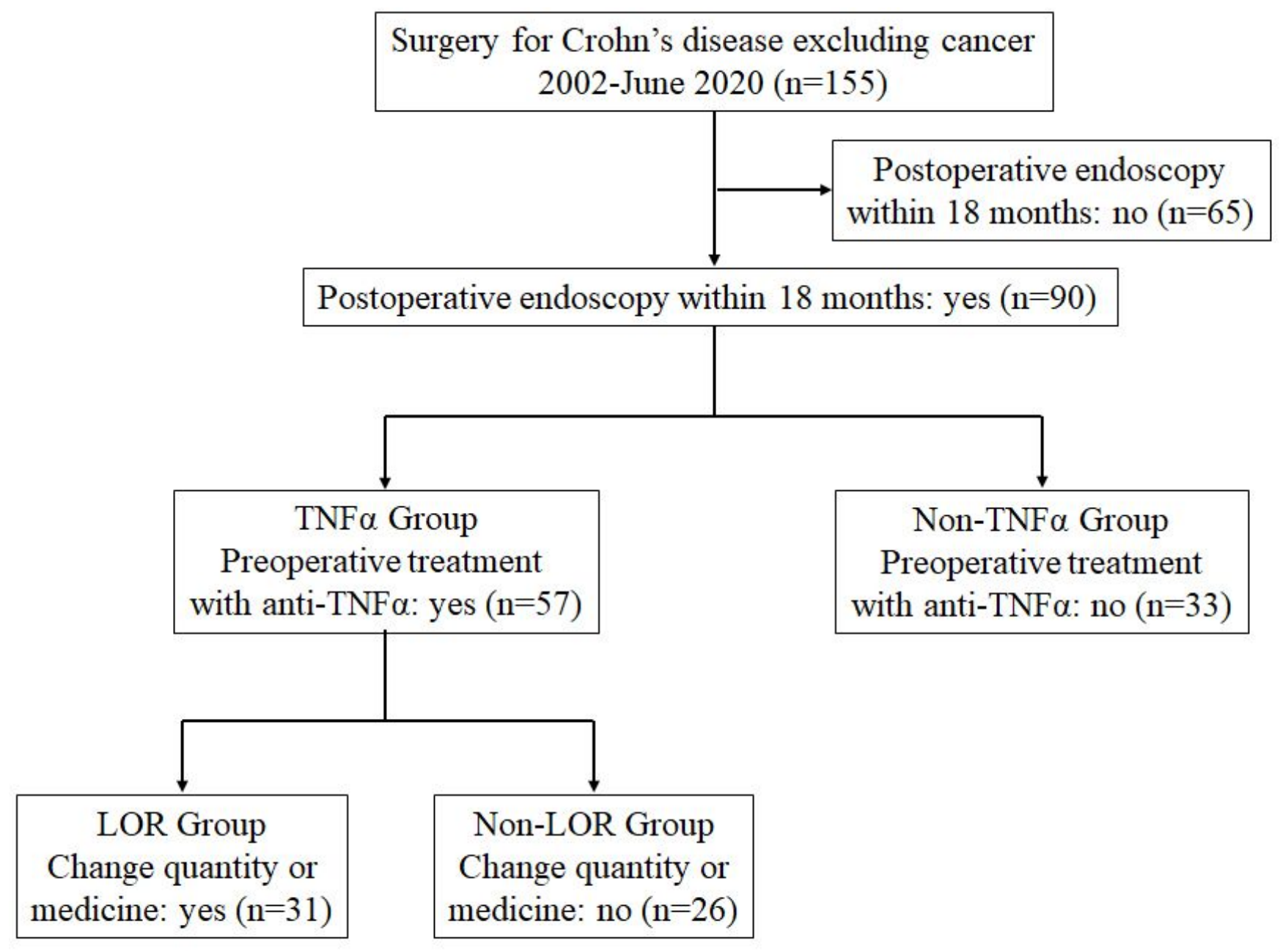

Figure 1

\section{Figure 1}

Patient selection. Anti-TNFa, anti-tumor necrosis factor $a$; LOR, loss of response 


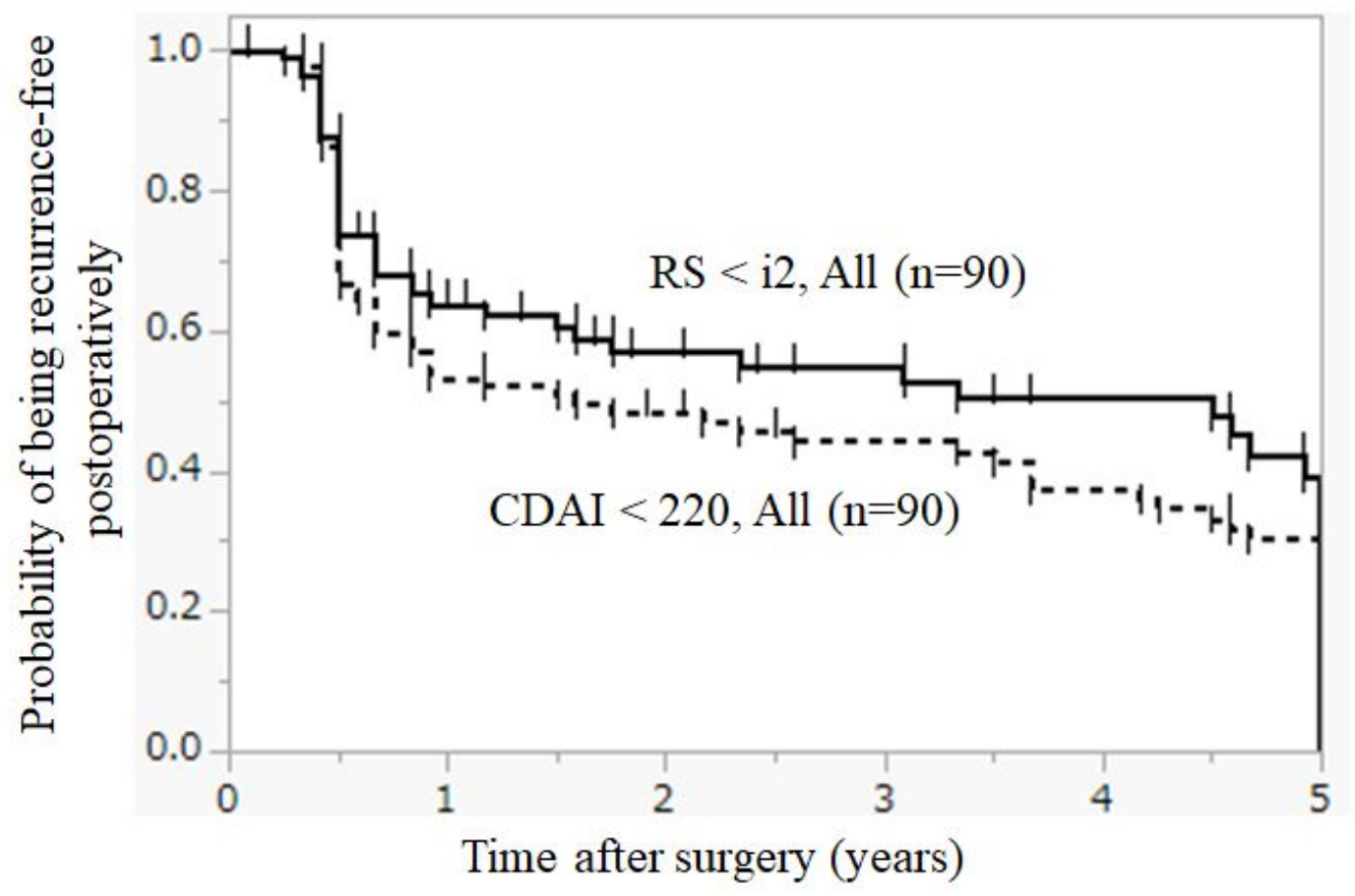

Endoscopic recurrence-free duration

........ Symptomatic recurrence-free duration

Figure 2

Figure 2

Postoperative symptomatic and endoscopic recurrence-free duration. The median symptomatic and endoscopic recurrence-free duration was 19 and 54 months. 

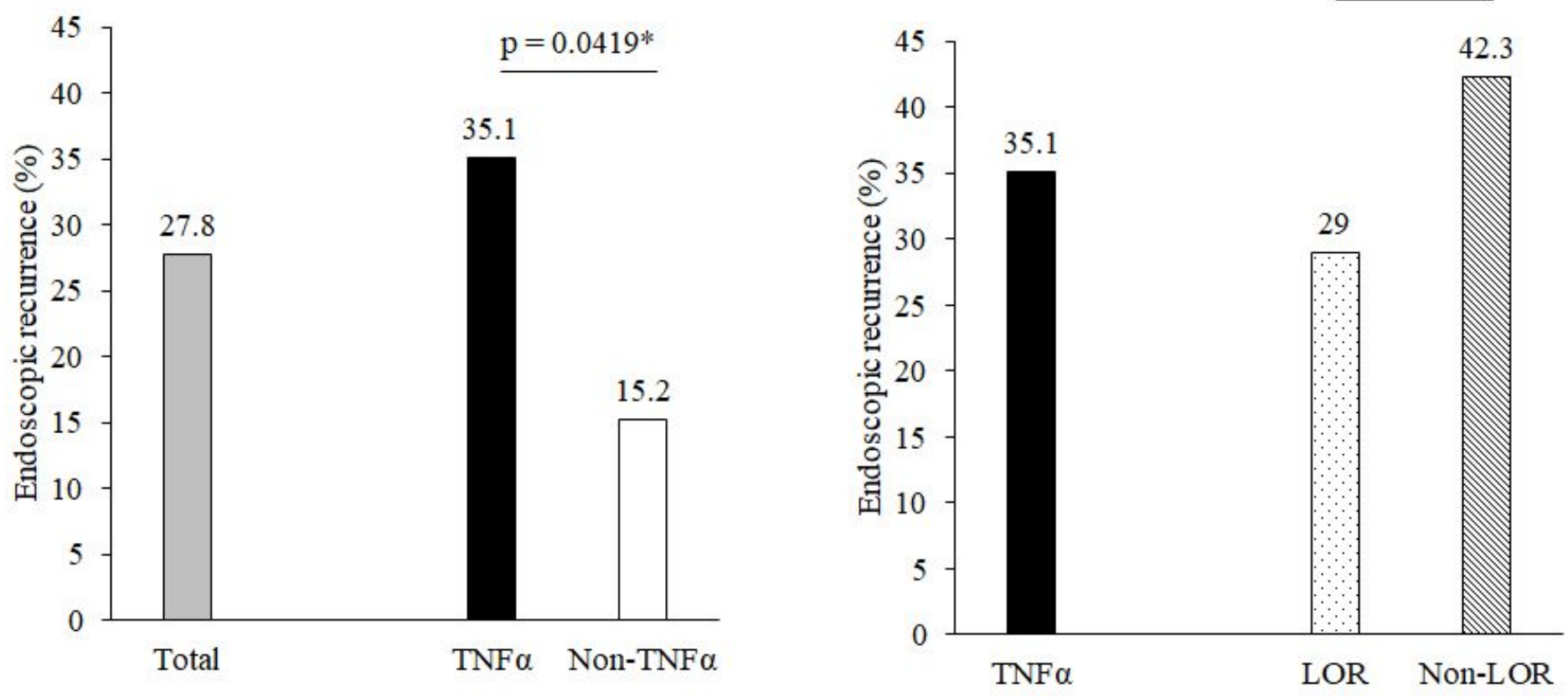

Figure 3

\section{Figure 3}

Rate of endoscopic recurrence at first postoperative endoscopy. The recurrence rate was significantly higher in the TNFa group than the non-TNFa group $(P=0.0419)$. 

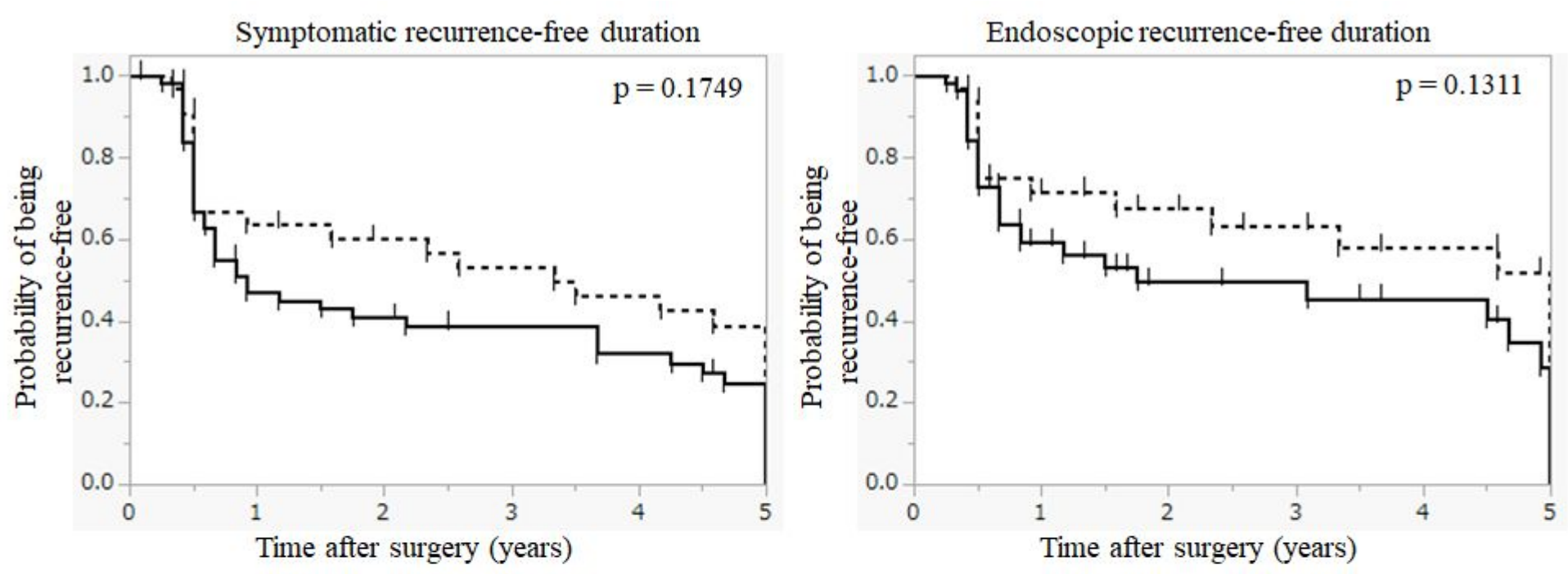

TNF $\alpha$ Group $(\mathrm{n}=57)$

Non-TNF $\alpha$ Group $(n=33)$

Figure 4

\section{Figure 4}

Symptomatic and endoscopic recurrence-free duration based on TNFa groups. 

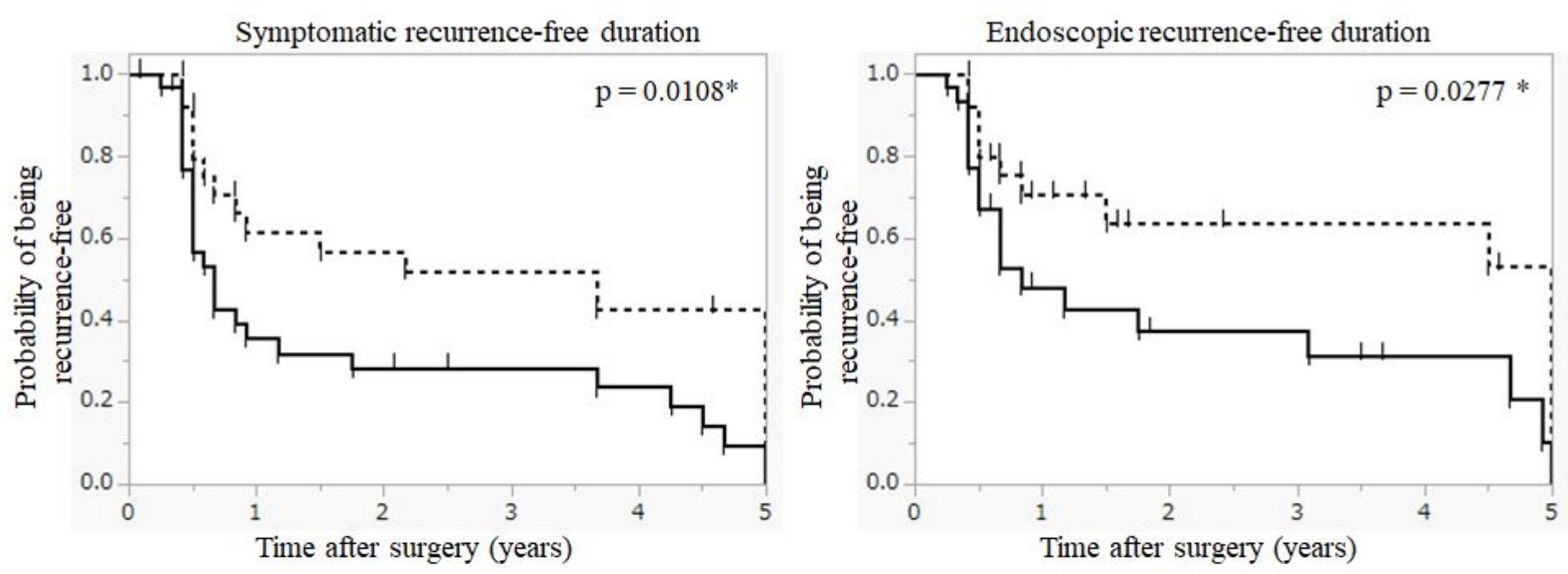

\section{LOR Group $(\mathrm{n}=31)$}

Non-LOR Group $(\mathrm{n}=26)$

Figure 5

\section{Figure 5}

Symptomatic and endoscopic recurrence-free duration based on LOR and non-LOR. 